\title{
Covid-19 pandemic: chronicle of responses and experiences of the infection prevention and control committee at a tertiary hospital in southwest Nigeria
}

\author{
Akinwumi Ayodeji Akinbodewa ${ }^{1}$, Michael Simidele Odimayo ${ }^{2}$, Olorunfemi Akinbode Ogundele, \\ Tosin Oluwapelumi Ogunleye ${ }^{4}$, Olanrewaju Olayinka Johnson ${ }^{4}$, Oluwakemi Abiola Lamidi ${ }^{5}$, \\ Mathew Akinmurele ${ }^{4}$, Oluwabunmi Motunrayo Oyebade ${ }^{6}$
}

1. Kidney Care Centre, department of Medicine, University of Medical Sciences Teaching Hospital, Ondo State, Nigeria.

2. Department of Microbial Pathology, University of Medical Sciences Teaching Hospital, Ondo State, Nigeria.

3. Department of Community Medicine, University of Medical Sciences Teaching Hospital, Ondo State, Nigeria.

4. Department of Nursing, University of Medical Sciences Teaching Hospital, Ondo State, Nigeria.

5. Department of Dietetics and Nutrition, University of Medical Sciences Teaching Hospital, Ondo State, Nigeria.

6. Department of Administration, University of Medical Sciences Teaching Hospital, Ondo State, Nigeria.

\section{Emails:}

ayoakinbodewa@yahoo.com;sodimayo@unimed.edu.ng; femidele@gmail.com; yemiluv2@gmail.com; olarex100@gmail.com; oluwakemi.lamidi@gmail.com; fineprincessgladys@gmail.com; angelina.bunmi77@ gmail.com

\begin{abstract}
Since the advent of 2019-Corona virus Disease (COVID-19) in Nigeria in February 2020, the number of confirmed cases has risen astronomically to over 61,307 cases within 8 months with more than 812 healthcare workers infected and some recorded deaths within their ranks.

Infection prevention and control is a key component in ensuring safety of healthcare workers in the hospital as healthcare-associated infection is one of the most common complications of healthcare management. Unbridled transmission of infection can lead to shortage of healthcare personnel, reduced system efficiency, increased morbidity and mortality among patients and in some instances, total collapse of healthcare delivery services. The Infection Prevention and Control Committee is a recognised group by the Centre for Disease Control and Prevention with their core programmes including drawing up activities, procedures and policies designed to achieve above-stated objectives before, during and after any disease outbreak, especially emerging and re-emerging ones such as the 2019 Coronavirus Disease. In this report, we highlight the roles played by the Infection Prevention and Control Committee of the University of Medical Sciences Teaching Hospital to prevent the spread of COVID-19 within and outside the hospital community and the lessons learned to date.
\end{abstract}

Keywords: COVID-19; infection prevention; infection control; Nigeria.

DOI: https://dx.doi.org/10.4314/ahs.v21i3.17

Cite as: Akinbodewa AA, Odimayo MS, Ogundele OA, Ogunleye TO, Johnson OO, Lamidi OA, et al. Covid-19 pandemic: chronicle of responses and experiences of the infection prevention and control committee at a tertiary hospital in southwest Nigeria. Afri Health Sci. 2021;21(3). 1093-1099. https://dx.doi.org/10.4314/abs.v21i3.17

\section{Corresponding author:}

Akinwumi Ayodeji Akinbodewa,

Department of Medicine

University of Medical Sciences Teaching Hospital

Ondo State, Nigeria.

Tel: +2348135641399

Email: ayoakinbodewa@yahoo.com
Healthcare-associated infection is one of the most common complications of healthcare management as it can lead to shortage of healthcare personnel, increased morbidity and mortality among patients and potential shut down of the health system; a situation which is made even more precarious during a pandemic. The 2019 Coronavirus Disease (COVID-19) has become a global pandemic with about 39.2 million people infect- 
ed in over 213 countries as of October 17, 2020 and more than 1.1 million deaths (case fatality rate: 0.03$).{ }^{1}$ The number of confirmed cases in Nigeria equally continues to rise and currently lies at 61,307 with 1,123 deaths since it was first reported in February in the country with a national case fatality rate of 0.02 . The cases are spread across all the thirty six States in Nigeria including Abuja, the Federal Capital Territory. ${ }^{2}$ Currently, Ondo State has recorded 1,654 cases with 36 deaths with a local case fatality rate of 0.02.2 More than 812 healthcare workers have tested positive for SARSCoV-2 with some recorded deaths nationwide. ${ }^{3}$

The University of Medical Sciences Teaching Hospital, Ondo State is a 472-bed tertiary centre established in 2018. Due to its status, it is frequently used as the last point of referral for critical cases from within and outside the State. Top government officials, high profile personalities and foreigners also access care at the hospital. Hence, it is natural that potential cases of COVID-19 will most likely present to the hospital; a history of recent travel to, or return from a COVID-19 prevalent country is one of the case definition criteia for the disease. In this report, we highlight the important roles of the Infection Prevention and Control Committee within and outside the hospital community during the current COVID-19 pandemic, modus operandi and lessons learned so far.

SARS-CoV-2 is a pleomorphic ribonucleic acid virus of the betacoronavirus genus. Its transmission by droplet and physical contact has been established with airborne spread linked only to aerosol generating medical procedures. However, newer evidence (which is largely experimental) has shown that airborne transmission of SARS-CoV-2 may be possible but this position has thrown open further debates considering the fact that its spread by airborne route is not consistent with viral particle size of $>5 \mu \mathrm{m}$ and its low viral reproduction number compared to viruses that are $\leq 5 \mu \mathrm{m}$ in size ( 2.5 v 18)..$^{4-5}$

The first step in SARS_CoV-2 infection is the interaction of its Spike (S) proteins with sensitive human cells. Genome encoding then occurs after entering into the cell and facilitates the expression of the genes that encode useful accessory proteins, which advance the adaptation of Coronaviruses to their human host. Virions are then released from the infected cell through exocytosis. The released viruses can proceed to infect kidney cells, liver cells, intestines and T lymphocytes, as well as the lower respiratory tract, where they form the main symptoms and signs. ${ }^{6-7}$

On-going efforts to develop a vaccine against SARS-
CoV-2 by various research groups have reached varying levels of development with twenty five candidate vaccines already at the level of clinical evaluation. ${ }^{8}$ The same can be said for drug development as there are ongoing studies to find the most effective $\operatorname{drug}(\mathrm{s}) /$ drug combination(s) for its cure. ${ }^{9}$ This unclear status of vaccine and drug development has left the fight against COVID-19 mainly at the level of prevention and control, at least for now.

The currently available options for the management of COVID-19 depends on whether the case is mild, moderate or severe.10 The World Health Organization recommends that all cases of COVID-19 should be isolated in order to contain viral transmission. Specifically, mild cases require mainly supportive care and symptomatic treatment (antipyretics, analgesics, adequate nutrition and hydration) while the more severe cases require in-hospital care, antibiotics (where indicated), close monitoring for disease progression, supplemental oxygenation when SPO2 is $<90 \%$. Those with Acute Respiratory Distress Syndrome are recommended to receive a trial of continuous positive airway pressure ventilation. The WHO has not recommended the use of choloroquine/hydroxychloroquine, antivirals, immunomodulators and plasma therapy for the treatment or prophylaxis of COVID-19 as existing published literature on these agents are mostly observational in nature with few clinical trials. ${ }^{10}$

\section{Composition of the Infection Prevention and Con- trol Committee}

Infection prevention and control (IPC) is a discipline that aims to prevent or control the spread of infections in healthcare facilities and the community. ${ }^{11}$ The primary goals of an IPC programme are to prevent susceptible patients acquiring disease-causing micro-organisms and to limit the spread of antimicrobial resistant infections. ${ }^{11-12}$ The Infection Prevention and Control Committee of the University of Medical Sciences Teaching Hospital was originally constituted in response to an upsurge in the outbreak of new cases of Lassa fever in the last quarter of 2019 in Ondo State with members drawn from various departments (Public Health, Microbiology, Internal Medicine, Medical Laboratory, Nursing, Dietetics, Administration, Public Relations and medical stores) primarily on volunteer basis. This was done to ensure that members were well-motivated personnel who were least occupied by other major responsibilities.

In order to ensure functional efficacy of the Committee members, series of formal training was provided to 
the team by the Local Government Medical Officer of Health, the Ondo State Epidemiologist and, the Ministry of Health in conjunction with Nigeria Centre for Disease Control (NCDC) under the NiCaDe (capacity development in training of infection prevention and control of health care workers at secondary and tertiary health care facilities) programme.

\section{UNIMEDTH IPC responses to COVID-19}

Our very first step was to conduct a needs assessment at the adult and paediatric Emergency rooms as well as the Obstetrics casualty room to determine their level of preparedness for the COVID-19 pandemic. This was followed by series of specialized training for healthcare workers deployed to these service points which included such modules as the nature of SARS-CoV-2, case definitions for COVID-19, importance of adequate history taking in differentiating between COVID-19 and other respiratory diseases, donning and doffing personal protective equipment and the appropriate communication channel for reporting suspected cases of COVID-19.

\section{Protocols and policies for managing COVID-19}

A number of protocols have been developed and updated by the Infection Prevention and Control Committee to address management of COVID-19 in the hospital one of which assists medical personnel on duty at the Emergency rooms (figure 1). The key areas of emphasis in the protocol included early involvement of the Consultant and adequate history taking to avoid confusing other respiratory illnesses with COVID-19. Studies have shown that senior physicians painstakingly reach a diagnosis while also requiring fewer investigations. ${ }^{13}$ Their involvement is also associated with better patient outcome while junior doctors have been known to have incomplete doctor-patient communication skills and significantly greater levels of anxiety due to uncertainty. ${ }^{13-15}$ These are less desirable qualities required during a pandemic where team stability and tight coordination are key requirements. It should also be noted that during the early period of COVID-19 outbreak in Wuhan, a well-tailored clinical evaluation chart was used to identify suspected cases and rule out potential differentials. ${ }^{16}$

\section{Doctor on duty to inform the Consultant/Senior Registrar immediately. Consultant to alert only the core managing team (doctors, nurses and}

support staff) and UNIMEDTH management. Avoid spreading panic.

2. Consultant to review history and coordinate care of suspected case UNTIL transfer to Infectious Disease Hospital (IDH) Akure.

3. Administer intranasal oxygen (where indicated) and order a chest x-ray. Consultant (or anyone so delegated) to draw samples for full blood count, thick and thin film for malaria parasite and serum chemistry at least. Please observe Universal Basic Precautions.

4. Transfer suspected cases within 30 minutes to the designated Isolation holding area (and not the Infectious Disease Hospital, Akure). Review patient with results of chest $\mathrm{x}$-ray and blood tests within 120 minutes of arrival.

5. Isolation unit is to provide cautious necessary care while awaiting review.

6. Where doctor on duty cannot reach the managing Consultant, please contact the respective Physician in Charge at Akure complex, Ondo complex or the DCMAC Clinical Services directly.

7. UNIMEDTH management to alert the Ondo State Infection Prevention and Control team.

8. Once confirmed positive, move patient at once to the Infectious Disease Hospital, Akure.

A production of the UNIMEDTH Infection Control and Prevention Committee

Contact us: call or WhatsApp (+234)08135641399, 07069307900, 08035154544, 08033871115

Figure 1: Updated Protocol for managing suspected cases of COVID-19 at University of Medical Sciences Teaching Hospital, Ondo State, Nigeria 
Nevertheless, caution must be applied in strictly relying on clinical evaluation in diagnosing and differentiating between COVID 19 and other respiratory illnesses nowadays as most countries are now officially at the stage of person-to-person transmission and many people (asymptomatic or not) with travel history and contact have not been forthcoming with information on their travel and contact history for fear of discrimination and stigmatization. ${ }^{17}$ Recently, in Nigeria, a number of cases who withheld relevant history have been treated inadvertently by healthcare workers. ${ }^{18-19}$

The protocol also emphasized carrying out investigations such as high resolution computerized tomography scan of the chest, full blood count and blood film for malaria parasite among others which could easily rule out common clinical differential diagnosis of COVID-19 in our setting. Employing this strategy has enabled us to triage suspected cases for immediate isolation while awaiting results of Reverse Transcriptase-Polymerase Chain Reaction of nasopharyngeal samples. Among over 40 Persons Under Investigation, 15 have been confirmed positive in our centre while others had diagnoses such as acute severe asthma, community acquired pneumonia, Chronic Obstructive Lung Diseases, non-cardiogenic pulmonary oedema of End Stage Renal Disease etc. This way, emergency needs of patientshave been addressed rather than focus on mounting pressure on the hospital management to "move patient away quickly" to the designated State Infectious Disease Hospital.

A protocol was also developed for the medical laboratory department for prevention of person-to-person transmission among staff. Recently, there have been claims that SARS-CoV-2 attaches to T-lymphocytes in the blood stream thus suggesting that the virus could be blood-borne..$^{20}$ Moreso, there have been questions as to how it is transported from the conjunctiva and other mucosa to the respiratory system.

While we have found these protocols useful in our local setting, they are not without their shortcomings. First is unwillingness of some medical staff to apply the protocols; some have even claimed ignorance of the protocols despite widespread circulation to all the wards, clinics, other service points and social media outlets. Secondly, there were senior nurses and physicians who, out of concern for their personal safety, chose to stay away from making contact with suspected cases thus leaving critical gaps in the execution of the protocols. These attitudes and behaviour have been linked to healthcare workers' discrimination against patients with highly infectious diseases. ${ }^{21-23}$

Due to limited financial support, we could not offer free or subsidized High Resolution Computerized Tomography scan to all suspected cases and most importantly, we experienced delay in receiving results of Reverse Transcriptase-Polymerase Chain Reaction tests of suspected cases for SARS-Cov-2, sometimes for up to 4 to 6 days thus exposing many of our patients, relatives and medical staff to undue psychological trauma.

The protocol for managing suspected cases of COVID-19 designed by our IPC team ${ }^{24}$ is an adaptation of the Centre for Disease Control guidelines bearing in mind stark realities of our limited human, capital and technological resources. It is not as elaborate as that used for hospitalised patients under investigation for Ebola viral disease in the United States of America where Patients Under Investigation were isolated in single rooms with fitted private bathrooms, and where there was continuous flow of Personal Protective Equipment for healthcare workers and dedicated medical equipment to care for the sick. ${ }^{25}$ It however aligns to a greater degree with the Centre for Disease Control protocol specifically designed for COVID-19 in terms of practice, Personal Protective Equipment use and reuse but still falls short in some other areas. ${ }^{26}$

\section{Medical advisories}

In order to ensure continuing medical education on COVID-19 for healthcare personnel, we have published regularly updated medical advisories for them (figure 2). A key element is the combination of cloth facemask and face shield especially with many countries already suggesting unconventional means of optimizing use of face masks and other essential Personal Protective Equipment in the face of dwindling resources. ${ }^{27}$

An advisory was also developed for non-clinical personnel working at the cash collection points. The main purpose of this was to protect them from possible transmission of the virus as paper money and coins could serve as potential vectors for transmission of COVID-19. While this is yet to be proven, a comprehensive review of earlier studies showed that bank notes and coins are capable of transmitting pathogens, even though these studies focused on bacteria, fungi and parasites, probably because most viruses do not survive long outside their host cells. ${ }^{28}$ Whether this could be applied to SARS-CoV-2 is yet to be elucidated as there is currently no available study in that direction but we do know that it survives for hours on different 
surfaces. In the meantime, the World Health Organization has advised that people should sanitize their hands after handling money. ${ }^{29}$ More importantly, we advised the Accounts department to promote electronic means of payment over cash transaction in order to eliminate or at best, reduce frequent contact with paper money.

1. Maintain a high index of suspicion when attending to all patients as many COVID-19 cases are asymptomatic. Ensure a distance of at least one metre from the patient.

2. Offer face masks to all patients who are coughing or sneezing. Take adequate history of recent travel or contact with a suspected/confirmed case from all patients.

3. Take a history of prior admission/treatment for respiratory illness (in UNIMEDTH or elsewhere) when attending to a patient with respiratory symptoms in order to rule out cases such as asthma, heart failure, chronic obstructive lung diseases, pulmonary tuberculosis, allergies etc.

4. Contact referring sister hospital (public or private) if you obtain history of recent treatment in such a facility (kindly notify hospital management). Seek assistance from a senior colleague when in doubt of diagnosis or next line of action.

5. Adhere to Universal Basic Precaution at all times when attending to all patients.

6. Use only antiseptic hand washing liquid soap; Say no to detergents or bar soap. Avoid diluting liquid soap with water. Instead, use moderate drops at every hand-washing session. It is more effective that way and, contrary to general assumption, more cost-effective.

7. Avoid wiping your hands with cloth (even your own) after washing; allow your hands to dry off in atmospheric air. Be careful not to soil the floor with drops of water.

8. Please note that when you have washed hands, there is no need to use hand sanitizer. Hand washing alone is as effective as using hand sanitizer. Use hand sanitizer before and after contact with every patient or their surrounding.

9. Avoid injudicious use of face masks. Adhere to strict indications for use of personal protective equipment (PPE). Kindly confer with your senior colleague for advice before donning Personal Protective Equipment.

10. Refer to the UNIMEDTH Handbook of Protocols for infection prevention and control regularly.

Committee

08035154544,08033871115
A production of the UNIMEDTH Infection Control and Prevention

Contact us: call or WhatsApp (+234)08135641399, 07069307900,

\section{Figure 2: Medical advisory to hospital staff on COVID-19 at University of Medical Sciences Teaching Hospital, Ondo State, Nigeria}

\section{Community engagement and enlightenment}

The IPC committee of the University of Medical Sciences Teaching Hospital works with the Disease Surveillance and Notification Officers, Red Cross Society, Environmental Officers and the traditional institutions as part of the Emergency Operation Committee to ensure infection prevention and control in the community. To achieve this during the COVID-19 pandemic, community-sponsored health-related radio Talk Shows were held in the local dialect and English language to reach a wide audience. For emphasis, hospital-sponsored banners bearing the message, "COVID-19 is real: stay home, stay safe" in both English and Yoruba languages were displayed around popular spots in both local governments.

\section{Local production of Personal Protective Equip-} ment and other hygienic materials

Standard guidelines for healthcare workers on hygienic practices have been released by the World Health Organization. ${ }^{30}$ However, similar to what is found in many other developing countries, these have not been easily achievable in our setting when compared to hospitals in developed countries. ${ }^{31}$

For example, hand washing, a key element in infection prevention and control has been difficult to comply with. The reasons for this include low level of awareness of infection control by healthcare staff and poor attitude, lack of time from heavy workload owing to shortage of healthcare work force, as well as lack of wash hand sinks and hygiene products. Secondly, hand disinfection (in between contact with patients) by means 
of alcohol-based hand sanitizers which takes less time and encourages higher compliance than hand washing is costly and so not always practicable due to limited resources. Other reasons for poor compliance to standard hygienic practices include poor and unsafe working environment, lack of running water and sinks as well as low (and often times irregular) remuneration. ${ }^{30}$ As part of continuing medical education for staff, the IPC committee produced a hospital-sponsored video on donning and doffing of PPE for regular group-viewing. The team was involved in identifying a suitable location for isolating and treating cases of COVID-19, production of locally sourced Personal Protective Equipment, methylated spirit and hand sanitizers.

\section{Conclusion}

The IPC committee's programmes during disease outbreak are essential tools to prevent or reduce infection spread among patients, healthcare workers and the community. The experience garnered so far from serving in the Committee indicates that so much more could be gained if hospitals strengthen their local Infection Prevention and Control Committees.

\section{Recommendations}

The IPC committee should be strengthened in all hospitals at all levels. While the leadership of an IPC team usually should be either a Microbial Pathologist, Community Physician or an Internist, membership should be mainly composed of well-motivated volunteers who are driven by altruism. Hospital managers should be willing to adequately support and fund their IPC committees.

\section{Limitations}

This is a report of a short-spanned, single centre experience.

\section{Conflict of interest}

No conflict of interest.

\section{References}

1. New cases of Cases of COVID-19 in world countries: daily confirmed new cases (5-day moving average). https://coronavirus.jhu.edu/data/new-cases. Accessed 18 October 2020.

2. COVID-19 Nigeria: confirmed cases by State. Downloaded from https://covid19.ncdc.gov.ng/ Accessed 18 October 2020.

3. Lara Adejoro. 812 healthcare workers infected with COVID-19 - NCDC. Downloaded from https:// healthwise.punchng.com/812-healthcare-workers-infected-with-covid-19-ncdc/. Accessed 30 July 2020.

4. Bourouiba L. Turbulent gas clouds and respiratory pathogen emissions: potential implications for reducing transmission of COVID-19. JAMA.2020;323(18):18371838.

5. Klompas M, Baker MA, Rhee C. Airborne transmission of SARS-CoV-2. Theoretical considerations and available evidence. JAMA. Published online July 13, 2020. doi:10.1001/jama.2020.12458.

6. Coronavirinae in Viral Zone. Available online: https://viralzone. expasy.org/785 Accessed 5 February 2019.

7. Lambeir AM, Durinx C, Scharpe S, De Meester I. Dipeptidylpeptidase IV from bench to bedside: An update on structural properties, functions, and clinical aspects of the enzyme DPP IV. Crit Rev Clin Lab Sci. 2003;40:209 -94 PubMed

8. DRAFT landscape of COVID-19 candidate vaccines - 28 July 2020. novel-coronavirus-landscape-covid-19cc0e97e4ea1b4458a05bbd6f5ac6d3fe.pdf. Accessed 30 July 2020

9. Nebehay S, Josephine Mason J. WHO sees first results from COVID drug trials within two weeks. https:// www.reuters.com/article/us-health-coronavirus-who/ who-sees-first-results-from-covid-drug-trials-withintwo-weeks-idUSKBN244219. Accessed 30 July 2020 10. WHO. Clinical management of COVID-19. Interim guidance 27 May 2020. Ref no: WHO/2019-nCov/clinical/2020.5. who.int/publications/i/item/clinical-management-of-covid-19. Accessed 18 October 2020.

11. Infection Prevention and Control guidelines. https://ncdc.gov.in/WriteReadData/1892s/File571. pdf. Accessed 22 April 2020.

12. Core infection prevention and control practices for safe healthcare delivery in all settings -recommendations of the healthcare infection control practices advisory committee. Last updated: March 15, 2017. https:/ / www.cdc.gov/hicpac/recommendations/core-practices.html. Accessed on April 22, 2020

13. Chao-Jui L, Yuan-Jhen S, Tsung-Cheng T, Kuan-Han W, Chien-Hung L, Yan-Ren L. The Impact of Emergency Physician Seniority on Clinical Efficiency, Emergency Department Resource Use, Patient Outcomes, and Disposition Accuracy, Medicine: February 2016;95(6): e2706 doi: 10.1097/MD.0000000000002706.

14. Canwell BM, Rarnirez AJ. Doctor-patient communication: a study of junior house officers. Med Educ. 1997; 31:17-21.

15. Bovier PA, Perneger TV. Stress from uncertainty 
from graduation to retirement: a population-based study of Swiss physicians. J Gen Intern Med. 2007; 22:632-638. 16. Zu ZY, Jiang MD, Xu PP, Chen W, Ni QQ, Lu GM et al. Coronavirus Disease 2019 (COVID19): A perspective from China. Radiology. 2020:200490. doi: 10.1148/ radiol.2020200490.

17. Wei L, Sha Z, Wang Y, Zhang G, Jia H, Zhou S et al. Willingness and cognition towards reporting travel history to high risk corona virus disease 2019 epidemic regions https://punchng.com/covid-19-kano-index-case-hid-travel-history-attended-parties-doctor/. among the Chinese public: a cross-sectional survey. BMC Public Health. 2020. DOI: 10.21203/rs.3.rs-22230/ v1.

18. COVID-19: Kano index case hid travel history, attended parties-Doctor. Accessed 24 April 2020.

19. Report: 55 year old man who failed to disclose travel history dies of COVID-19 at Lagos University Teaching Hospital. https://www.thecable.ng/report-55-yearold-man-who-failed-to-disclose-travel-history-dies-ofcovid-19-at-luth. Accessed 24 April 2020.

20. Wang X, Xu W, Hu G, Xhia S, Sun Z, Liu Z et al. SARS-CoV-2 infects $T$ lymphocytes through its spike protein-mediated membrane fusion. Cell Mol Immunol. 2020. https://doi.org/10.1038/s41423-020-0424-9.

21. Akinbodewa AA, Ige OO, Gbala MO, Akinbodewa GO. COVID-19 related patient discrimination by healthcare workers: a single centre experience. Indian Journal of Case Reports. 2020;6(5): 224-226.

22. Bharat S, Aggleton P, Tyrer P. India: HIV and AIDS-Related Discrimination, Stigmatization and Denial. Geneva: Joint United Nations Programme on HIV/AIDS; 2001. p. 16-7. Available from: http://www. data.unaids.org/Publications/IRC-pub02/JC587-India_en.pdf. [Last accessed on 2020 Apr 09].

23. Narasimhulu DM, Edwards V, Chazotte C, Bhatt D, Weedon J, Minkoff H. Healthcare workers' at- titudes toward patients with ebola virus disease in the United States. Open Forum Infect Dis. 2016;3:1-7. PubMed 24. COVID-19: Handbook of protocols for infection prevention and control at the University of Medical Sciences Teaching Hospital, September 2020. ISBN number: 978-978-57821-3-4. www.researchgate.net. Accessed 18 October 2020.

25. Infection Prevention and Control Recommendations for Hospitalized Patients Under Investigation (PUIs) for Ebola Virus Disease (EVD) in U.S. Hospitals. https://www.cdc.gov/vhf/ebola/clinicians/evd/ infection-control.html. Accessed 30 July 2020.

26. Interim infection prevention and control recommendations for healthcare personnel during the coronavirus disease 2019 (COVID-19) pandemic. Updated July 15, 2020. https://www.cdc.gov/coronavirus/2019-ncov/ hcp/infection-control recommendations.html?CDC_ AA_refVal=https $\% 3 \mathrm{~A} \% 2 \mathrm{~F} \% 2 \mathrm{Fwww}$.cdc.gov $\% 2 \mathrm{~F}$ coronavirus $\% 2 \mathrm{~F} 2019$-ncov $\% 2$ Finfection-control $\% 2 \mathrm{~F}$ control-recommendations.html. Accessed 31 July 2020. 27. Livingston E, Desai A, Berkwits M. Sourcing personal protective equipment during the COVID-19 Pandemic. JAMA Published online March 28, 2020. doi:10.1001/jama.2020.5317.

28. Angelakis E, Azhar EI, Bibi F, Yasir M, Al-Ghamdi AK, Ashshi AM et al. Paper money and coins as potential vectors of transmissible disease. Future microbiol. 2014;9(2): 249-61

29. The WHO has clarified that they aren't warning people against using paper money due to coronavirus.https: / / fullfact.org/health/coronavirus-WHO-cash-comments/. Accessed 25 April 2020. 30. WHO. Prevention of hospital-acquired infections: A practical guide. Geneva: WHO; 2002

31. Lynch P, Pittet D, Borg MA, Mehtar S. Infection control in countries with limited resources. J Hosp Infect. 2007;65 Suppl 2:148-50. 